\title{
Effects of Ipragliflozin on Postprandial Glucose Metabolism and Gut Peptides in Type 2 Diabetes: A Pilot Study
}

\author{
Hiroaki Ueno (D) Hiroko Nakazato · Emi Ebihara - Kenji Noma • \\ Takahisa Kawano $\cdot$ Kazuhiro Nagamine $\cdot$ Hideyuki Sakoda • \\ Masamitsu Nakazato
}

Received: November 10, 2017 / Published online: January 10, 2018

(c) The Author(s) 2018. This article is an open access publication

\section{ABSTRACT}

Introduction: Ipragliflozin is a novel antidiabetic drug that inhibits renal tubular sodiumglucose cotransporter-2 (SGLT2). The aim of this study was to evaluate the effects of ipragliflozin on glucose, insulin, glucagon, and gastrointestinal peptide responses to a meal tolerance test, as well as to investigate the glucose-lowering mechanisms of ipragliflozin.

Methods: Nine Japanese patients with obesity and type 2 diabetes mellitus were treated with ipragliflozin ( $50 \mathrm{mg} /$ day) for 12 weeks. The postprandial profiles of glucose, insulin, glucagon, active glucagon-like peptide-1 (GLP-1), active glucose-dependent insulinotropic polypeptide (GIP), ghrelin, and des-acyl ghrelin

Enhanced content To view enhanced content for this article go to http://www.medengine.com/Redeem/ A02DF060212BE85F.

H. Ueno $(\varangle) \cdot$ H. Nakazato · E. Ebihara

K. Nagamine $\cdot$ H. Sakoda $\cdot$ M. Nakazato

Division of Neurology, Respirology, Endocrinology and Metabolism, Department of Internal Medicine,

Faculty of Medicine, University of Miyazaki,

Miyazaki, Japan

e-mail: intron@med.miyazaki-u.ac.jp

K. Noma

Noma Naika Clinic, Miyazaki, Japan

T. Kawano

Kawano Naika Clinic, Miyazaki, Japan were measured before and 12 weeks after ipragliflozin treatment.

Results: Body weight, body fat mass, systolic blood pressure, and HbA1c and serum uric acid levels were significantly decreased after the treatment. Postprandial glucose and insulin levels were also significantly decreased. Postprandial glucagon increased both before and after ipragliflozin treatment; however, the increment tended to be smaller after treatment. Active GLP-1, active GIP, ghrelin, and des-acyl ghrelin did not change after treatment.

Conclusion: Ipragliflozin improved glycemic control by reducing body weight, postprandial inappropriate glucagon secretion, and the postprandial insulin requirement. Although this was a short-term study with a small sample size, ipragliflozin may offer benefits for patients with obesity and type 2 diabetes mellitus.

Trial Registration: University Hospital Medical Information Network (UMIN No. 000017195).

Keywords: Ghrelin; GLP-1; Glucagon; Ipragliflozin; Type 2 diabetes mellitus

\section{INTRODUCTION}

Individuals with obesity are at high risk of developing diabetes mellitus. In addition, after developing diabetes mellitus, their glycemic control easily worsens. 
These individuals are more likely to develop complications such as dyslipidemia, hypertension, and hepatic steatosis. Conversely, when obese diabetic patients lose $3-5 \%$ or more of their weight, they benefit from improvements in these complications [1].

Developed countries have an overabundance of inexpensive, high-calorie foods and advanced transportation systems; thus, individuals living in these countries may have difficulty reducing their energy consumption and adhering to lifestyle changes to their diets or exercise therapy.

Sodium-glucose cotransporter-2 (SGLT2) inhibitors suppress the activity of SGLT2 expressed in renal tubules and increase urinary glucose excretion, thereby improving blood glucose levels [2]. In Japan, SGLT2 inhibitors became available in 2015; in most obese diabetic patients, these drugs help to decrease body weight and improve HbA1c levels [3]. A doubleblind comparative study of an SGLT2 inhibitor, empagliflozin, in diabetic patients (including Asian patients) who were at high risk of cardiovascular events showed that empagliflozin significantly reduced total mortality and the incidence of cardiovascular events [4-6].

The mechanisms underlying the weight loss and reduction of plasma glucose level using SGLT2 inhibitors are believed to occur through loss of glucose in the urine. However, it is possible that these medications may work through other mechanisms such as gastrointestinal peptides and glucagon. The effects of SGLT2 inhibitors on gastrointestinal peptides and glucagon in diabetic patients are not fully clarified. We hypothesized that ipragliflozin may affect the secretion of gastrointestinal peptides and glucagon to improve plasma glucose levels and to reduce body weight, at least in part. Therefore, we examined the glucose metabolism as well as gastrointestinal peptides before and after ipragliflozin treatment in this study. This study aimed to investigate the effects of an SGLT2 inhibitor, ipragliflozin, including its effects on glucose metabolism. Ipragliflozin was administered for 12 weeks to nine patients with obesity and type 2 diabetes mellitus. Meal tolerance tests (MTTs) were performed before and after ipragliflozin administration to study changes in glucose metabolism and gastrointestinal hormones, including glucagon-like peptide-1 (GLP-1), glucose-dependent insulinotropic polypeptide (GIP), glucagon, ghrelin, and des-acyl ghrelin.

\section{METHODS}

\section{Subjects}

We conducted an open-label study to investigate the effects of $50 \mathrm{mg} /$ day of ipragliflozin in patients with type 2 diabetes mellitus. The patients ranged in age from 20 to 70 years, with HbA1c levels between $6.0 \%$ and $9.0 \%$. The patients were recruited between August 2015 and October 2016 from three study sites: Miyazaki University Hospital, Noma Naika clinic, and Kawano Naika clinic. We excluded patients with any of the following conditions: patients with an estimated glomerular filtration rate of $45 \mathrm{~mL} / \mathrm{min} / 1.73 \mathrm{~m}^{2}$, patients with severe hepatic function disorders or malignant tumors, patients with severe microvascular or macrovascular complications, pregnant patients, patients already receiving oral administration of SGLT2 inhibitors, patients with allergies to SGLT2 inhibitors, and patients receiving insulin or GLP-1 receptor agonists. Of the 11 patients who were enrolled in the study, nine patients (seven men and two women) were included in the analysis; the two excluded patients dropped out voluntarily. Patient characteristics are shown in Table 1.

\section{Study Protocol}

All patients provided written consent for participation in this study. We instructed them to visit Miyazaki University Hospital after fasting for at least $10 \mathrm{~h}$ and measured their height, weight, body composition (InBody 720; InBody Japan Inc.), blood pressure, and pulse rate. Subsequently, MTTs (426 kcal; carbohydrates $59.7 \%$; protein $9.5 \%$; lipids $30.7 \%$ ) were performed. Blood samples were taken at 0 (baseline), 15, 30, 45, 60, 90, 120, and $180 \mathrm{~min}$ postprandial; blood glucose, insulin, glucagon, active GLP-1, active GIP, ghrelin, and des-acyl 
Table 1 Baseline characteristics of subjects

\begin{tabular}{ll}
\hline Characteristics & Value \\
\hline Age (years) & $50.0 \pm 8.0$ \\
Sex (men/women) & $7 / 2$ \\
Body mass index $\left(\mathrm{kg} / \mathrm{m}^{2}\right)$ & $29.8 \pm 4.4$ \\
HbAlc $(\%)$ & $7.2 \pm 0.6$ \\
Fasting blood glucose $(\mathrm{mg} / \mathrm{dL})$ & $139.9 \pm 17.7$ \\
Duration of T2DM (year) & $4.9 \pm 4.7$ \\
Hypertension/dyslipidemia & $3 / 7$ \\
Treatment & \\
Diet only & 4 \\
Metformin & 5 \\
DPP-4 inhibitors & 3 \\
Sulfonylureas & 1 \\
Pioglitazone & 1 \\
Number of oral hypoglycemic drugs $1 / 2 / 3$ & $1 / 3 / 1$ \\
\hline
\end{tabular}

$T 2 D M$ type 2 diabetes mellitus, DPP-4 dipeptidyl peptidase -4

ghrelin levels were measured at all blood sampling times. Blood glucose and insulin levels were measured with a hexokinase assay and a chemiluminescent enzyme immunoassay (SRL Inc., Japan), respectively. For measurements of blood active GLP-1, active GIP, and glucagon, blood was collected using P-800 blood collection tubes (Japan Becton, Dickinson and Company, Japan); the tubes were centrifuged for $15 \mathrm{~min}$ at $3000 \mathrm{rpm}$ and $4{ }^{\circ} \mathrm{C}$ to isolate plasma. The samples for glucagon measurements were immediately placed in a freezer at $-70{ }^{\circ} \mathrm{C}$, then glucagon levels were measured by a sandwich enzyme-linked immunosorbent assay (ELISA) using $\mathrm{N}$ - and C-terminal specific antibodies (Mercodia AB, Sweden).

The samples for active GLP- 1 and active GIP measurements were extracted using OASIS HLB (Nihon Waters K.K., Japan). Active GLP-1 and active GIP levels were measured with the active GLP-1 ELISA kit (Millipore, Billerica, MA, USA) and the active GIP ELISA kit (Yanaihara Inc.,
Japan), respectively. To measure ghrelin and des-acyl ghrelin levels, blood was collected directly into tubes containing aprotinin; the tubes were immediately centrifuged for plasma isolation at $4{ }^{\circ} \mathrm{C}$. The isolated plasma was treated with one-tenth of its volume of $1 \mathrm{~N} \mathrm{HCl}$ and the tubes were rocked gently. These samples were then used for plasma ghrelin and des-acyl ghrelin measurements using an automated enzyme immunoassay (AIA-600II, Tosoh Corporation, Japan) as described elsewhere [7]. Baseline levels of serum lipids, liver functions, renal functions, malondialdehyde (MDA)-modified low-density lipoprotein (LDL) as oxidized LDL, 8-hydroxy-2'-deoxyguanosine (8-OHdG) as a marker of oxidative stress, high-sensitivity C-reactive protein (hs-CRP) as an inflammatory marker, adiponectin, and leptin were measured (SRL Inc., Japan). The day after MTT, patients started to receive $50 \mathrm{mg}$ of ipragliflozin (once daily in the morning) concurrently with the oral drugs they had been receiving prior to the tests; the patients continued to make outpatient visits. MTTs were performed again 12 weeks after ipragliflozin administration.

This study was approved by the Ethics Review Committee of the University of Miyazaki and is registered in the University Hospital Medical Information Network Clinical Trials Registry as UMIN000017195. All procedures followed were in accordance with the ethical standards of the responsible committee on human experimentation (institutional and national) and with the Helsinki Declaration of 1964, as revised in 2013.

\section{Statistical Analysis}

All results are presented as mean \pm standard deviation. Statistical analysis was performed by means of appropriate parametric and nonparametric methods.

Changes in continuous measures of laboratory test values before and 12 weeks after treatment were analyzed using paired $t$ tests. Nonparametric methods were used for non-normally distributed values. Repeated-measures analysis of variance models were used to analyze the glucose, insulin, glucagon, active GLP- 
1, active GIP, ghrelin, and des-acyl ghrelin mean response profiles over the eight-time point MTT. JMP 12.0 (SAS Institute Inc., Cary, NC, USA) and PRISM 5.0 (GraphPad Software Inc., La Jolla, CA, USA) were used for statistical analysis. $P$ values less than 0.05 were considered statistically significant.

\section{RESULTS}

Changes in body weight, body composition, blood pressure levels, and pulse rates are shown in Table 2. Twelve weeks after the commencement of ipragliflozin administration, body weight significantly decreased, with a mean weight loss of $2.7 \mathrm{~kg}$. Body fat mass and ratios significantly decreased, whereas no significant changes were noted in skeletal muscle mass or quantity of body water. Systolic blood pressure significantly decreased, with diastolic blood pressure levels showing a decreasing tendency $(P=0.058)$.

HbA1c levels were reduced by $0.4 \%$. MTT revealed that overall blood glucose levels and its area under the curve (AUC) significantly improved after ipragliflozin treatment, and

Table 2 Changes in the parameters after 12-week ipragliflozin treatment

\begin{tabular}{lccl}
\hline & 0 weeks & 12 weeks & P value \\
\hline Body weight $(\mathrm{kg})$ & $82.0 \pm 13.9$ & $79.3 \pm 12.6$ & $0.024^{*}$ \\
BMI $\left(\mathrm{kg} / \mathrm{m}^{2}\right)$ & $29.8 \pm 4.4$ & $28.6 \pm 4.4$ & $0.023^{*}$ \\
Fat mass $(\mathrm{kg})$ & $27.6 \pm 10.0$ & $25.4 \pm 10.2$ & $0.009^{*}$ \\
Muscle mass $(\mathrm{kg})$ & $30.2 \pm 5.3$ & $30.3 \pm 5.4$ & 0.640 \\
Body water $(\mathrm{kg})$ & $39.6 \pm 6.3$ & $39.8 \pm 6.4$ & 0.623 \\
\% body fat & $33.3 \pm 8.5$ & $31.3 \pm 9.5$ & $0.005^{*}$ \\
SBP (mmHg) & $138.6 \pm 12.9$ & $131.7 \pm 13.9$ & $0.042^{*}$ \\
DBP (mmHg) & $88.3 \pm 8.6$ & $80.3 \pm 12.1$ & 0.058 \\
Pulse rate $(/ \mathrm{min})$ & $93.2 \pm 14.1$ & $90.7 \pm 9.8$ & 0.451 \\
\hline
\end{tabular}

$B M I$ body mass index, $S B P$ systolic blood pressure, $D B P$ diastolic blood pressure

${ }^{*} P<0.05$ glucose levels at all time points during postprandial $120 \mathrm{~min}$ significantly decreased (Fig. 1a, b). Overall insulin levels and AUC significantly decreased after ipragliflozin treatment (Fig. 1c, d). Plasma glucagon increased postprandially and then slowly decreased before ipragliflozin treatment; however, after the treatment, overall plasma glucagon levels tended to be lower $(P=0.096)$ (Fig. 1e). There was no significant difference in glucagon AUC until 180 min after MTT, but the AUC calculated by the results until the first $60 \mathrm{~min}$ was significantly lower with ipragliflozin treatment (Fig. 1f). Comparisons of active GLP-1, active GIP, ghrelin, and des-acyl ghrelin levels before and after ipragliflozin treatment revealed no statistical differences (Fig. 1g-k). Plasma ghrelin level at $180 \mathrm{~min}$ tended to increase from the fasting level in the 12 weeks after ipragliflozin treatment compared to before treatment $(\Delta 0-180 \mathrm{~min}$ of ghrelin: $-1.5 \pm 3.9$ before treatment vs. $2.5 \pm 4.7 \mathrm{pmol} / \mathrm{L}$ after treatment, $P=0.063$ ).

Changes in blood parameters before and after ipragliflozin treatment are shown in Table 3. Acetoacetic acid showed an increasing tendency $(P=0.06)$; however, no differences were noted in 3-hydroxybutyric acid or total ketone bodies. No significant changes in serum lipids (LDL cholesterol, high-density lipoprotein cholesterol, and triglycerides) or liver functions were noted. No changes were noted in renal functions; however, uric acid significantly decreased. No changes were noted in urinary protein excretion, although urinary glucose excretion significantly increased. There were no significant changes in the homeostatic model assessment for insulin resistance, leptin, adiponectin, hs-CRP, MDA-LDL, or 8-OHdG. No noteworthy adverse reactions occurred during this study.

\section{DISCUSSION}

In the present study, a 12-week administration of ipragliflozin to obese Japanese patients with type 2 diabetes mellitus resulted in the improvement of blood glucose levels and body weight. Blood pressure and uric acid levels also 
A
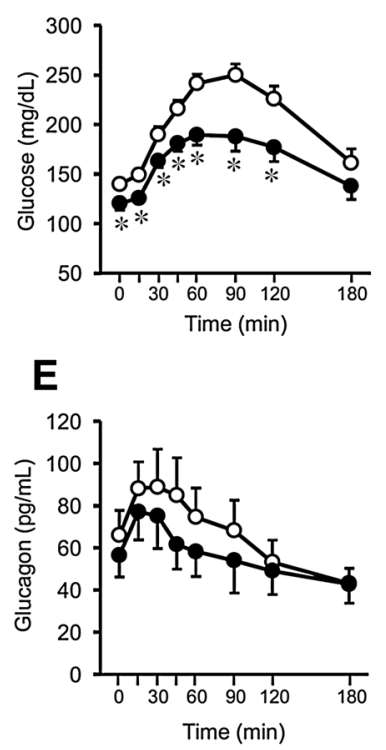

I

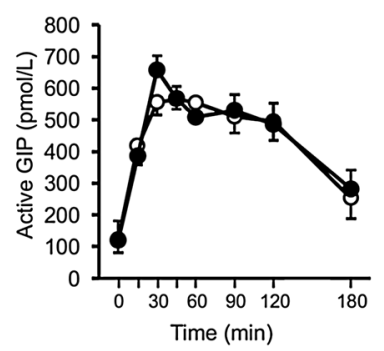

B

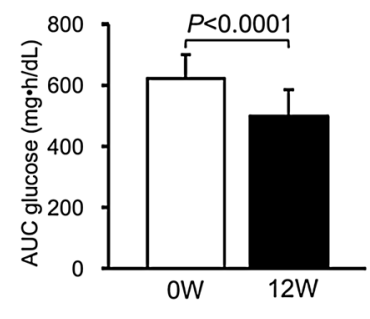

$\mathbf{F}$

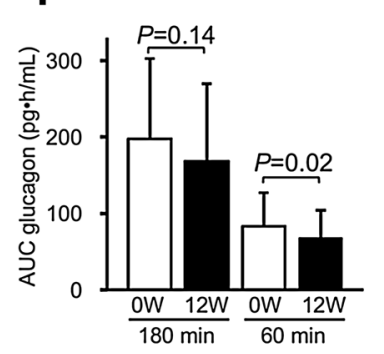

J

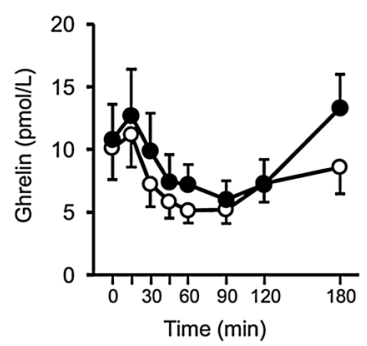

Fig. 1 Effects of ipragliflozin on glucose metabolism and gut peptides in the meal tolerance test. The changes are shown for a plasma glucose (before vs. after, $P<0.0001$ ), b glucose $\mathrm{AUC}_{0-180}$, $\mathbf{c}$ insulin (before vs. after, $P=0.042$ ), d insulin $\mathrm{AUC}_{0-180}$, e plasma glucagon (before vs. after, $P=0.096)$, f glucagon $\mathrm{AUC}_{0-180}$ and $\mathrm{AUC}_{0-60}$, $\mathbf{g}$ active GLP-1 (before vs. after, $P=0.28$ ), $\mathbf{h}$ active GLP-1

improved. Although the levels of active GLP-1, active GIP, ghrelin, and des-acyl ghrelin did not change after treatment, postprandial increment of glucagon levels tended to be smaller after treatment.

SGLT2 inhibitors are thought to improve blood glucose levels by promoting glucosuria [8]. Changes in plasma glucagon and insulin levels are also seen after using SGLT2 inhibitors, but the underlying mechanisms are not fully understood. In our study, postprandial insulin levels significantly decreased after ipragliflozin treatment. Previous studies described
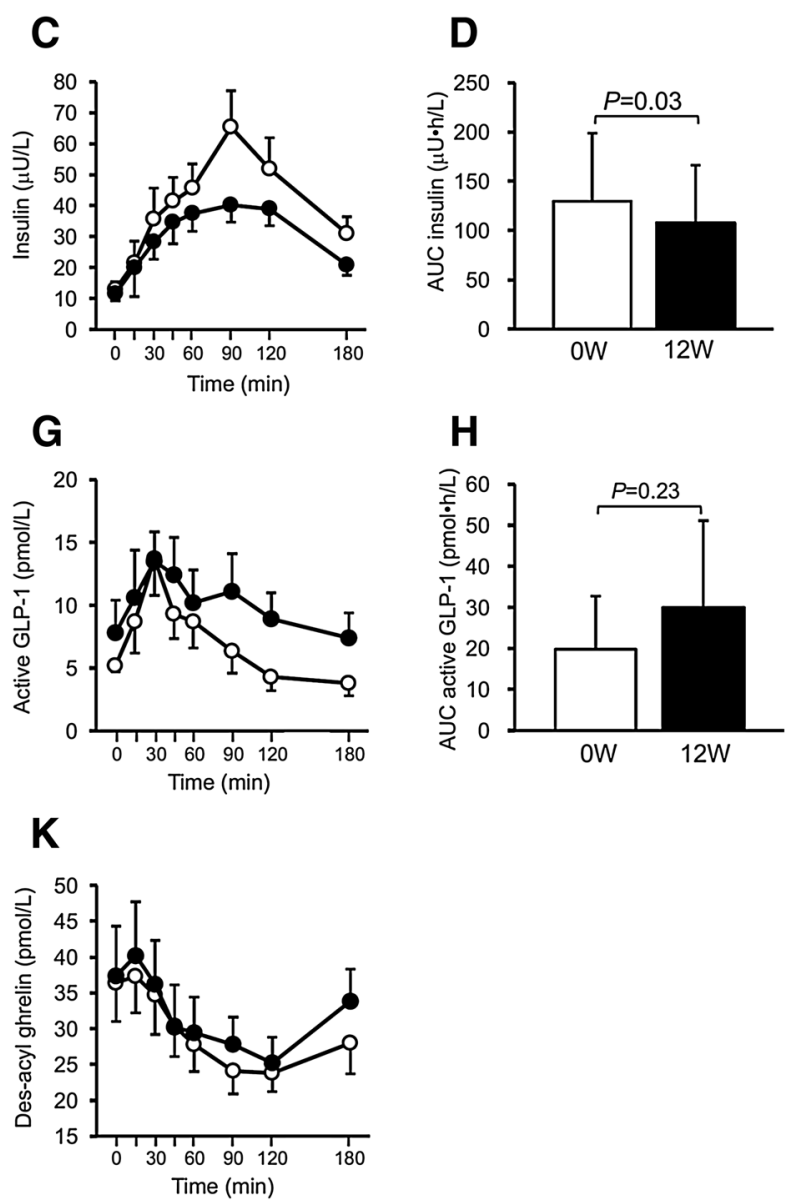

AUC $_{0-180}$, $\mathbf{i}$ active GIP (before vs. after, $P=0.78$ ), j ghrelin (before vs. after, $P=0.35$ ), and $\mathbf{k}$ des-acyl ghrelin (before vs. after, $P=0.28$ ). Open and closed circles indicate before and after 12 weeks of ipragliflozin administration, respectively. ${ }^{*} P<0.05$ vs. before ipragliflozin administration

decreasing levels of both plasma glucose and insulin levels after SGLT2 inhibitor treatment in diabetic patients [9]. Because SGLT2 inhibitors do not directly affect either beta cell function or tissue glucose utilization, the decrease in serum insulin levels is regarded as a secondary effect of decreasing body weight and plasma glucose levels [10].

In our study, postprandial glucagon secretion tended to decrease after ipragliflozin treatment. Several previous studies reported that treatment with an SGLT2 inhibitor (dapagliflozin, luseogliflozin, or empagliflozin) for 
Table 3 Changes in the parameters after 12-week ipragliflozin treatment

\begin{tabular}{|c|c|c|c|}
\hline & 0 weeks & 12 weeks & $P$ value \\
\hline HbAlc (\%) & $7.2 \pm 0.6$ & $6.8 \pm 0.3$ & $0.03^{*}$ \\
\hline BUN (mg/dL) & $14.1 \pm 3.0$ & $15.3 \pm 3.3$ & 0.26 \\
\hline Cre $(\mathrm{mg} / \mathrm{dL})$ & $0.70 \pm 0.16$ & $0.70 \pm 0.17$ & 0.83 \\
\hline $\mathrm{eGFR}\left(\mathrm{ml} / \mathrm{min} / 1.73 \mathrm{~m}^{2}\right)$ & $89.7 \pm 15.8$ & $91.2 \pm 18.1$ & 0.41 \\
\hline Uric acid $(\mathrm{mg} / \mathrm{dL})$ & $6.2 \pm 1.5$ & $5.0 \pm 1.2$ & $0.003^{*}$ \\
\hline $\operatorname{AST}(\mathrm{IU} / \mathrm{L})$ & $24.9 \pm 9.1$ & $26.9 \pm 12.5$ & 0.34 \\
\hline $\operatorname{ALT}(\mathrm{IU} / \mathrm{L})$ & $32.0 \pm 16.3$ & $34.1 \pm 22.6$ & 0.58 \\
\hline$\gamma-\mathrm{GTP}(\mathrm{IU} / \mathrm{L})$ & $45.2 \pm 21.0$ & $36.6 \pm 14.0$ & 0.28 \\
\hline LDL-cho $(\mathrm{mg} / \mathrm{dL})$ & $123.0 \pm 39.3$ & $126.6 \pm 24.1$ & 0.64 \\
\hline HDL-cho $(\mathrm{mg} / \mathrm{dL})$ & $45.8 \pm 9.2$ & $47.1 \pm 15.2$ & 0.65 \\
\hline Triglyceride (mg/dL) & $182.2 \pm 40.0$ & $176.9 \pm 51.9$ & 0.79 \\
\hline HOMA-R & $4.4 \pm 2.5$ & $3.5 \pm 2.2$ & 0.13 \\
\hline UPCR (mg/g.Cre) & $86.1 \pm 92.9$ & $95.0 \pm 65.2$ & 0.64 \\
\hline UGCR (mg/g·Cre) & $73.6 \pm 27.2$ & $25,106 \pm 21,591$ & $0.008^{*}$ \\
\hline Acetoacetic acid (mmol/L) & $2.8 \pm 1.2$ & $7.7 \pm 8.4$ & 0.06 \\
\hline 3-Hydroxybutyric acid $(\mu \mathrm{mol} / \mathrm{L})$ & $111.7 \pm 94.4$ & $179.1 \pm 244.3$ & 0.42 \\
\hline Total ketone body $(\mu \mathrm{mol} / \mathrm{L})$ & $113.4 \pm 95.1$ & $186.4 \pm 251.7$ & 0.40 \\
\hline Adiponectin $(\mu \mathrm{g} / \mathrm{ml})$ & $6.0 \pm 3.0$ & $6.0 \pm 2.7$ & 1.0 \\
\hline Leptin $(\mathrm{ng} / \mathrm{mL})$ & $19.0 \pm 16.0$ & $16.5 \pm 12.5$ & 0.24 \\
\hline hs-CRP (ng/mL) & $1700 \pm 1559$ & $1830 \pm 1683$ & 0.56 \\
\hline MDA-LDL (U/L) & $162.0 \pm 45.8$ & $167.6 \pm 47.1$ & 0.43 \\
\hline 8-OHdG (ng/mL) & $0.14 \pm 0.03$ & $0.12 \pm 0.02$ & 0.22 \\
\hline
\end{tabular}

UPCR urinary protein to creatinine ratio, $U G C R$ urinary glucose to creatinine ratio, $h s$-CRP high sensitivity C-reactive protein, $M D A$ - $L D L$ malondialdehyde-modified low-density lipoprotein, 8-OHdG 8-hydroxy-2'-deoxyguanosine

${ }^{*} P<0.05$

1-12 weeks significantly increased fasting or postprandial plasma glucagon levels in humans [9, 11-15]. However, two other articles reported no changes in fasting or postprandial plasma glucagon levels after ipragliflozin or dapagliflozin treatment in humans [16, 17]. Although the reason for this discrepancy in results between the present study and previous studies is unknown, it may be related to the glucagon measuring methods used. Of the eight previous studies mentioned above, four studies lacked a description of their glucagon measuring methods $[11,13,14,17]$, and the remaining four used radioimmunoassay (RIA) for glucagon measurement $[9,12,15,16]$. Glucagon in the blood rapidly degrades after blood sampling, thus requiring a blood sampling tube containing a protease inhibitor and prompt centrifugation after blood sampling. Furthermore, because RIA uses an antibody that recognizes 
the C-terminal of glucagon, the possibility of measuring proglucagon-derived peptides other than glucagon cannot be ruled out. Although sandwich ELISA kits can be used for a glucagon assay at present, some ELISA kits have problems related to specificity, precision, and sensitivity [18]; the kit manufactured by Mercodia used in our study is reported to have the best performance [19]. Because we measured glucagon with the most reliable sandwich ELISA kit and appropriate blood sampling, the plasma glucagon levels recorded in our study are closer to the actual levels. Although inhibition of inappropriate postprandial glucagon secretion after ipragliflozin treatment may be one of the mechanisms of plasma glucose improvement, the sample size of our study was too small to arrive at such a conclusion; thus further studies using appropriate measuring methods are still needed.

SGLT2 inhibitors resulted in increased food consumption in mice $[20,21]$. Furthermore, in human studies, SGLT2 inhibitor treatment increased the amount of food intake as calculated with a mathematical model [22]. In the present study, overall plasma ghrelin levels in MTT did not change after ipragliflozin treatment for 12 weeks. Considering that basal plasma ghrelin levels were inversely correlated with body mass index in diabetic patients in our previous study [23], plasma ghrelin levels might increase because of a body weight reduction following SGLT2 inhibitor treatment. The tendency for postprandial elevation of plasma ghrelin may be one of the explanations for the appetite gain observed after SGLT2 inhibitor treatment. This also requires further study.

The major limitation of this study was its small sample size; other limitations include uncontrolled observational study design, insufficient observation period, and analysis of body composition using the impedance method (by which changes in visceral fat and subcutaneous fat remain unknown).

\section{CONCLUSIONS}

Twelve-week treatment with ipragliflozin in Japanese obese patients with type 2 diabetes mellitus resulted in improved glycemic control, which allowed for a reduction in insulin requirements. Improvements in body weight, blood pressure, and serum uric acid levels were also observed. Furthermore, postprandial excessive glucagon secretion tended to decrease. However, this was an observational study with a small sample size; thus, further studies are needed.

\section{ACKNOWLEDGEMENTS}

Funding. This study and article processing charges were supported by a Grant for Clinical Research from Miyazaki University Hospital.

Authorship. All named authors meet the International Committee of Medical Journal Editors (ICMJE) criteria for authorship for this manuscript, take responsibility for the integrity of the work as a whole, and have given final approval for the version to be published.

Disclosures. Hiroaki Ueno, Hiroko Nakazato, Emi Ebihara, Kenji Noma, Takahisa Kawano, Kazuhiro Nagamine, Hideyuki Sakoda, and Masamitsu Nakazato have nothing to disclose.

Compliance with Ethics Guidelines. All procedures followed were in accordance with the ethical standards of the responsible committee on human experimentation (institutional and national) and with the Helsinki Declaration of 1964, as revised in 2013. Informed consent was obtained from all patients for being included in the study. The study protocol was approved by the Ethical Committee of University of Miyazaki (no. 2014-044). 
Data Availability. The datasets used in the current study are available from the corresponding author on reasonable request.

Open Access. This article is distributed under the terms of the Creative Commons Attribution-NonCommercial 4.0 International License (http://creativecommons.org/licenses/ by-nc/4.0/), which permits any noncommercial use, distribution, and reproduction in any medium, provided you give appropriate credit to the original author(s) and the source, provide a link to the Creative Commons license, and indicate if changes were made.

\section{REFERENCES}

1. Muramoto A, Matsushita M, Kato A, et al. Three percent weight reduction is the minimum requirement to improve health hazards in obese and overweight people in Japan. Obes Res Clin Pract. 2014;8:e466-75.

2. Gomez-Peralta F, Abreu C, Lecube A, et al. Practical approach to initiating SGLT2 inhibitors in type 2 diabetes. Diabetes Ther. 2017;8:953-62.

3. Zaccardi F, Webb DR, Htike ZZ, et al. Efficacy and safety of sodium-glucose co-transporter- 2 inhibitors in type 2 diabetes mellitus: systematic review and network meta-analysis. Diabetes Obes Metab. 2016;18:783-94.

4. Kaku K, Lee J, Mattheus M, et al. Empagliflozin and cardiovascular outcomes in Asian patients with type 2 diabetes and established cardiovascular disease-results from EMPA-REG OUTCOME. Circ J. 2017;81:227-34.

5. Zinman B, Wanner C, Lachin JM, et al. Empagliflozin, cardiovascular outcomes, and mortality in type 2 diabetes. N Engl J Med. 2015;373:2117-28.

6. Anderson JE, Wright EE Jr, Shaefer CF Jr. Empagliflozin: role in treatment options for patients with type 2 diabetes mellitus. Diabetes Ther. 2017;8:33-53.

7. Tsuchimochi $\mathrm{W}$, Ueno $\mathrm{H}$, Yamashita $\mathrm{E}$, et al. Teneligliptin improves glycemic control with the reduction of postprandial insulin requirement in Japanese diabetic patients. Endocr J. 2015;62:13-20.
8. Kalra S. Sodium glucose co-transporter-2 (SGLT2) inhibitors: a review of their basic and clinical pharmacology. Diabetes Ther. 2014;5:355-66.

9. Ferrannini E, Muscelli E, Frascerra S, et al. Metabolic response to sodium-glucose cotransporter 2 inhibition in type 2 diabetic patients. J Clin Invest. 2014;124:499-508.

10. Kaneto H, Obata A, Kimura $\mathrm{T}$, et al. Beneficial effects of sodium-glucose cotransporter 2 inhibitors for preservation of pancreatic beta-cell function and reduction of insulin resistance. J Diabetes. 2017;9:219-25.

11. Okamoto A, Yokokawa H, Sanada H, et al. Changes in levels of biomarkers associated with adipocyte function and insulin and glucagon kinetics during treatment with dapagliflozin among obese type 2 diabetes mellitus patients. Drugs $\mathrm{R} D$. 2016;16:255-61.

12. Nishimura R, Osonoi T, Kanada $\mathrm{S}$, et al. Effects of luseogliflozin, a sodium-glucose co-transporter 2 inhibitor, on 24-h glucose variability assessed by continuous glucose monitoring in Japanese patients with type 2 diabetes mellitus: a randomized, double-blind, placebo-controlled, crossover study. Diabetes Obes Metab. 2015;17:800-4.

13. Nishimura R, Omiya H, Sugio K, et al. Sodium-glucose cotransporter 2 inhibitor luseogliflozin improves glycaemic control, assessed by continuous glucose monitoring, even on a low-carbohydrate diet. Diabetes Obes Metab. 2016;18:702-6.

14. Al Jobori H, Daniele G, Adams J, et al. Determinants of the increase in ketone concentration during SGLT2 inhibition in NGT, IFG and T2DM patients. Diabetes Obes Metab. 2017;19:809-13.

15. Merovci A, Solis-Herrera C, Daniele G, et al. Dapagliflozin improves muscle insulin sensitivity but enhances endogenous glucose production. J Clin Invest. 2014;124:509-14.

16. Hansen L, Iqbal N, Ekholm E, et al. Postprandial dynamics of plasma glucose, insulin, and glucagon in patients with type 2 diabetes treated with saxagliptin plus dapagliflozin add-on to metformin therapy. Endocr Pract. 2014;20:1187-97.

17. Ishihara H, Yamaguchi S, Nakao I, et al. Efficacy and safety of ipragliflozin as add-on therapy to insulin in Japanese patients with type 2 diabetes mellitus (IOLITE): a multi-centre, randomized, placebocontrolled, double-blind study. Diabetes Obes Metab. 2016;18:1207-16.

18. Bak MJ, Albrechtsen NW, Pedersen J, et al. Specificity and sensitivity of commercially available assays for glucagon and oxyntomodulin 
measurement in humans. Eur $\mathrm{J}$ Endocrinol. 2014;170:529-38.

19. Wewer Albrechtsen NJ, Hartmann B, Veedfald S, et al. Hyperglucagonaemia analysed by glucagon sandwich ELISA: nonspecific interference or truly elevated levels? Diabetologia. 2014;57:1919-26.

20. Vallon V, Gerasimova M, Rose MA, et al. SGLT2 inhibitor empagliflozin reduces renal growth and albuminuria in proportion to hyperglycemia and prevents glomerular hyperfiltration in diabetic Akita mice. Am J Physiol Renal Physiol. 2014;306:F194-204.

21. Devenny JJ, Godonis HE, Harvey SJ, et al. Weight loss induced by chronic dapagliflozin treatment is attenuated by compensatory hyperphagia in dietinduced obese (DIO) rats. Obesity. 2012;20:1645-52.

22. Ferrannini G, Hach T, Crowe S, et al. Energy balance after sodium-glucose cotransporter 2 inhibition. Diabetes Care. 2015;38:1730-5.

23. Ueno H, Shiiya T, Mizuta M, et al. Plasma ghrelin concentrations in different clinical stages of diabetic complications and glycemic control in Japanese diabetics. Endocr J. 2007;54:895-902. 